\title{
Space charge limited conduction in CdSe thin films ${ }^{\dagger}$
}

\author{
PRADIP KR. KALITA*, B K SARMA and H L DAS \\ Department of Physics, Gauhati University, Guwahati 781 014, India \\ *Department of Physics, Guwahati College, Guwahati 781 021, India
}

MS received 16 November 2002; revised 7 July 2003

\begin{abstract}
The current $(I)$-voltage $(V)$ characteristics of thermally evaporated CdSe thin films having thickness in the range 850-3000 $\AA$ and deposited within the substrate temperature of 303-573 K show nearly linear dependence at low voltage and afterwards a non-linear behaviour at higher voltage range. A detailed study of $I-V$ curves in dark and under illumination clearly reveals the mechanism as ohmic at low voltage and that of trap limited space charge limited conduction (SCLC) at higher voltage. The transition voltage $\left(V_{t}\right)$ from ohmic to SCLC is found to be quite independent of ambient temperature as well as intensity of illumination. SCLC is explained on the basis of the exponential trap distribution in CdSe films. Trap depths estimated from the In $I$ vs $10^{3} / T$ plots are found to be within $0 \cdot 60-0 \cdot 37 \mathrm{eV}$. Using the relevant SCLC theory, the carrier concentration, $n_{0}$, total trap concentration, $N_{t}$, and the ratio of free charge to trapped charge, $\theta$, have been calculated and correlated with ambient temperature and intensity of illumination.
\end{abstract}

Keywords. CdSe thin film; $I-V$ characteristics; SCLC mechanism; localized traps.

\section{Introduction}

CdSe has emerged as a promising material in recent years because of its potential technological importance. It is widely preferred in fabrication of solar cells, photodetector, light emitting diodes and other opto-electronic devices owing to its high photosensitive behaviour compared to other II-VI materials (Sathyalatha et al 1989; Padmanabhasarma et al 1995). CdSe often possesses $n$-type conductivity in bulk as well as in thin film form (Oduor and Gould 1995). The native defects in excess Cd and Se vacancies thus play an important role in the determination of conduction processes in CdSe thin films (Raoult et al 1989; Oduor and Gould 1995; Nesheva 1996).

The current $(I)$-voltage $(V)$ characteristics in thin films is an important tool for analysing the different conduction processes. Several workers (Gogoi and Barua 1982; Gould and Ismail 1992) studied the $I-V$ curves in II-IV semiconductor films with a variety of methods and thereby determined the various physical processes viz. schottky, Pool-Frankel and space charge limited conduction depending on the film growth and experimental ambient conditions. However, the work done so far in CdSe thin films in order to characterize the defect levels and their influence on the physical processes is insufficient for a better understanding of conduction mechanism. Most of the

\footnotetext{
*Author for correspondence

'The paper was presented at the 6th Asian Thermo Physical Properties Conference, October 2001, Gauhati University, Guwahati
}

investigations were carried out using sandwich type films compared to a lesser amount of work done using gap type films. Moreover, the gap type film configuration is more favourable for direct photoresponse study. Keeping in view of above aspects, SCL conduction process based on the experimental $I-V$ characteristics in dark and under illumination in thermally evaported CdSe films has been analysed and reported in this paper.

\section{Experimental}

Thin films of cadmium selenide were deposited on glass substrates by usual thermal evaporation technique (HINDHIVAC $12 \mathrm{~A} 4$ ) at a vacuum $\sim 10^{-6}$ torr. High purity (99.999\%) bulk CdSe powder obtained from Koch Light Lab, UK was used for deposition of films at substrate temperature $\left(T_{\mathrm{s}}\right)$ in the range $303-573 \mathrm{~K}$. The film thickness $(t)$ was measured with the help of a multiple beam interferometry method. Gap type film geometry with a effective gap of $3 \mathrm{~mm}$ between the electrodes was made to facilitate photoresponse study. Pure $\mathrm{Al}, \mathrm{Ag}$ and In were taken for preparation of electrode films.

The current measurement was done by using a high impedance $\left(\sim 10^{14} \Omega\right)$ ECIL electrometer amplifier with an accuracy of $3 \%$. The film was kept inside a continuously evacuated glass chamber which along with other measuring equipments was again kept inside a shielding network in the form of a Farady Cage during measurements. A $250 \mathrm{~W}$ quartz halogen bulb was used for white light illumination and the intensity of illumination was measured with the help of a sensitive Aplab luxmeter. 


\section{Results and discussion}

\subsection{I-V characteristics}

Study on $I-V$ characteristics is a matter of importance for properly analysing the conduction mechanism in films. A non-linear curve often may reveal the existence of different kinds of conduction mechanisms. In the present work the non-linear $I-V$ characteristics of the films are examined and explained on the basis of SCL conduction. The $I-V$ relationship shows an overall non-linear behaviour within the applied bias $-160 \mathrm{~V}-160 \mathrm{~V}$. The electrode-film contacts were examined with pre-evaporated $\mathrm{Ag}, \mathrm{Al}$, and In films which show identical non-linear behaviour and thereby rule out the possibility of electrode effect. Non-linearity is thus the manifestation of volume conductivity that can explore the feasibility of different conduction mechanisms (Khare et al 2000). The $I-V$ characteristics in $\ln -\ln$ scale for different CdSe thin films deposited at $T_{\mathrm{s}}$ within $303-573 \mathrm{~K}$ are depicted in figure 1 . Another plot of $I-V$ curves measured from $303-$ $393 \mathrm{~K}$ for a film deposited at $423 \mathrm{~K}$ having thickness, $3000 \AA$, is shown in figure 2. Figure 3 represents the same characteristic of a film in dark and under different white light illuminations. Figures $1-3$ clearly show two distinct slopes for each of the curves within the applied bias.

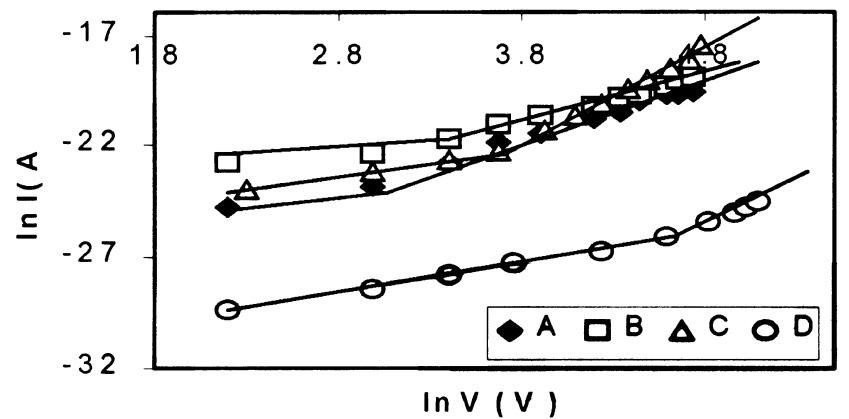

Figure 1. $\ln I-\ln V$ characteristics of four CdSe films. A: $850 \AA(t), 303 \mathrm{~K}\left(T_{\mathrm{s}}\right)$; B: $1400 \AA, 473 \mathrm{~K}$; C: $2000 \AA$, $573 \mathrm{~K}$ and D: $3000 \AA, 423 \mathrm{~K}$.

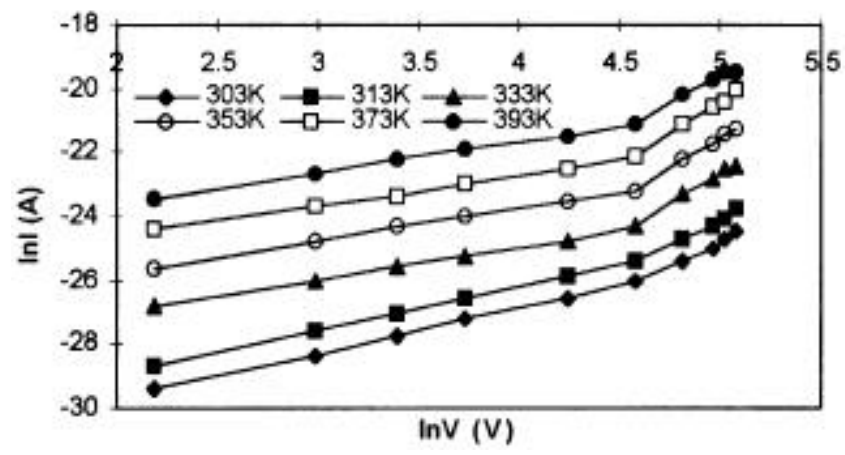

Figure 2. $\ln I-\ln V$ characteristics for a CdSe film (D) measured at $303-393 \mathrm{~K}$.
For lower applied bias the value of slopes are 1.25, 0.80, 1.25 and 1.38 whereas for higher bias the slopes change to $2.5,2 \cdot 8,3.8$ and 3.0 as shown in figure 1 for four $\mathrm{CdSe}$ films having thicknesses $850 \AA, 1400 \AA, 2000 \AA$ and $3000 \AA$, respectively. Similar behaviour of changing slope with the increasing bias is also found when the current measurement is done at different ambient temperatures and white light illuminations (figures 2 and 3 ). Thus the $I-V$ curves show two regions of conduction, viz. (i) nearly ohmic at lower bias and (ii) non-ohmic conduction at higher bias, in these $\mathrm{CdSe}$ films. For lower bias the injected charge carrier density is lower than the thermally generated carrier density and that leads to ohmic behaviour. At higher bias, as the slopes are $>2$ it clearly suggests that the conduction in this region is dominated by trap limited space charge limited conduction mechanism (Devi and Prakash 1992, 1993; Oduor and Gould 1995; Goswami 1996). The transition voltage $\left(V_{\mathrm{t}}\right)$ at which the transition occurs from ohmic to SCLC is expected to depend on the film growth condition. It is evident from figure 1 that $V_{\mathrm{t}}$ changes with the change in film thickness as well as substrate temperature. However, it is found that $V_{\mathrm{t}}$ is quite invariant on increasing ambient temperature and intensity of illuminations. Similar characteristics of SCLC in II-VI films is also reported by other workers (Lhermitte et al 1975; El-Kadry et al 1995; Nesheva 1996).

\subsection{Conduction mechanism}

The conduction mechanism in compound thin films is mainly governed by the grain boundary defect states (Pawlikowski 1990; Kalita et al 1999). Native defects and their concentration is dependent on the film growth condition. Thus the defect states which effectively act as either trapping or recombination centres play an important role in determination of conduction mechanism in the films deposited at different growth conditions. At lower bias up to transition voltage $\left(V_{\mathrm{t}}\right)$, the current density $(J)$ governed by the intrinsic free carrier density $\left(n_{0}\right)$, in the film can be expressed as

$$
J=n_{0} \mu v_{t} / d,
$$

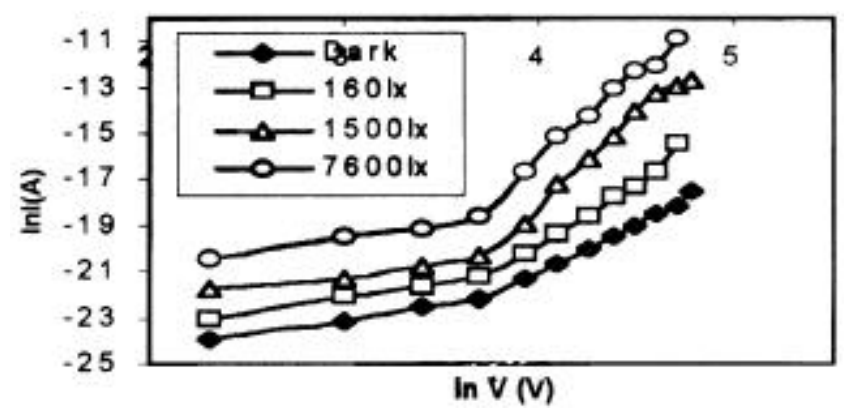

Figure 3. $\ln I-\ln V$ characteristics for a CdSe film (C) in dark and under white light illuminations. 
here $d$ is the gap between the electrodes and $\mu$ the electron mobility. It is established that CdSe in bulk and in thin film form always possesses $n$-type conductivity. Therefore, the hole transport is taken to be at minimum.

In the high bias region usually a slope of 2 indicates shallow trapping. However, in the present case as the slopes are $>2$, it clearly suggests that the SCLC is governed by the exponential trap distribution preferably with deep traps. Therefore, this trap limited SCLC current density is expressed as (Oduor and Gould 1995),

$$
J=e \mu N_{\mathrm{c}}\left(\varepsilon / e N_{0} k T_{l}\right)^{l} v^{l+1} / d^{2 l+1},
$$

here $N_{0}$ is the trap density per unit energy range at conduction band edge, $\varepsilon$ the permittivity of the film taken as 5.76 (Sharma et al 1992) and $l$ the ratio of $\left(T_{l} / T\right)$, here $T_{l}$ and $T$ are the characteristic temperature (Bak and Lipinski 1994) of energetic trap distribution and ambient temperature, respectively. Other parameters have their usual significance. The total trap concentration is given by

$$
N_{\mathrm{t}}=N_{0} k T_{l} \text {. }
$$

The injected carrier density becomes greater than the free carrier density at high bias which leads to SCL conductions in the films. At transition voltage $\left(V_{\mathrm{t}}\right)$ where conduction changes from ohmic to SCLC, the injected carrier density is equal to free carrier density. Therefore, at transition voltage, $n_{0}$ is the measurement of normal volume generated free carrier density (Khare et al 2000). Assuming the earlier reported value of $\mu=2.2 \times 10^{-8} \mathrm{~m}^{2} \mathrm{v}^{-1} \mathrm{~s}^{-1}$ (Buragohain and Barua 1987) measured within ambient temperatures, 303-423 K and under illuminations, 3500$7000 \mathrm{~lx}$ for gap type CdSe film, $n_{0}$ is calculated from relation (1). Equating (1), (2) and (3), the total trap concentration, $N_{\mathrm{t}}$, is determined as

$$
N_{\mathrm{t}}=\left(N_{\mathrm{c}} / n_{0}\right)^{1 / 1}\left(\varepsilon v_{\mathrm{t}} / e d^{2}\right)
$$

Here $N_{\mathrm{c}}$ is the effective density of states in the conduction band given by the relation

$$
N_{\mathrm{c}}=2\left(2 \pi m_{\mathrm{e}} k T / h^{2}\right)^{3 / 2},
$$

here the symbols have their usual meanings. $m_{\mathrm{e}}$ is the effective electron mass taken as $0.13 \mathrm{~m}_{0}$ for CdSe films (Oduor and Gould 1995). $N_{c}$ increases with ambient temperature. Using (4) the corresponding density of total trap concentration is determined. If $\theta$ be the fraction of total charge injected being available for conduction then it can be approximately related to $n_{0}$ as (Khare et al 2000)

$$
n_{0}=\left(\varepsilon \varepsilon_{0} \theta v_{\mathrm{t}} / e d^{2}\right)
$$

Thus using (6) $\theta$ can be estimated. The trap depth, $E_{\mathrm{t}}$, is a function of $\theta$ which can be expressed as

$$
\theta=\left(N_{\mathrm{c}} / N_{\mathrm{t}}\right)\left(\exp \left(-E_{\mathrm{t}} / k T\right)\right)
$$

A plot of $\ln I$ vs $10^{3} / T$ in the SCLC region thus can measure the trap depths (Goswami 1996). Figure 4 shows $\ln I$ vs $10^{3} / T$ plots for a representative CdSe thin film when the bias voltage are applied above transition voltage. All curves either measured in dark or under white light illumination have a single slope each and the slope decreases with increasing bias and intensity of illumination. The trap depths corresponding to slopes for different $\mathrm{CdSe}$ films are calculated which are found to be within 0.60$0.37 \mathrm{eV}$. These trap depths are in conformity with the earlier reported works (Yodogawa et al 1973; Raoult et al 1989; Sathyalatha et al 1989). The photocurrent measured in the same film shows a sublinear relationship with the intensity of illumination (figure 5). Hence it supports the defect controlled SCLC dominated by those deep trapping states.

The calculated values of free carrier density, $n_{0}$, total trap density, $N_{\mathrm{t}}$, ratio of injected charge to trapped charge, $\theta$, and the trap depths are systematically represented in tables 1 and 2. It is seen that the fraction of free carriers for conduction $(\theta)$ and the corresponding free carrier density increases with increasing ambient temperature and intensity of illumination where the total trap density decreases. This is predominantly due to thermal ionization and minority carrier recombination with the trapping centres. In pure compound film like $\mathrm{CdSe}$, the grain boundary core contains a large number of defects due to

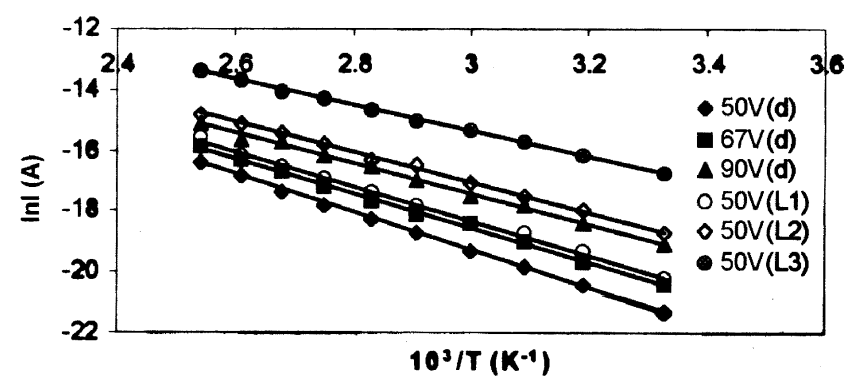

Figure 4. $\ln I-\ln 10^{3} / T$ plots for CdSe film (C) measured in dark (d) and under illuminations $\left(L_{1}: 160 \mathrm{~lx} ; L_{2}: 1500 \mathrm{~lx}\right.$ and $L_{3}$ : $7600 \mathrm{~lx})$. Biases are applied above $V_{\mathrm{t}}$.

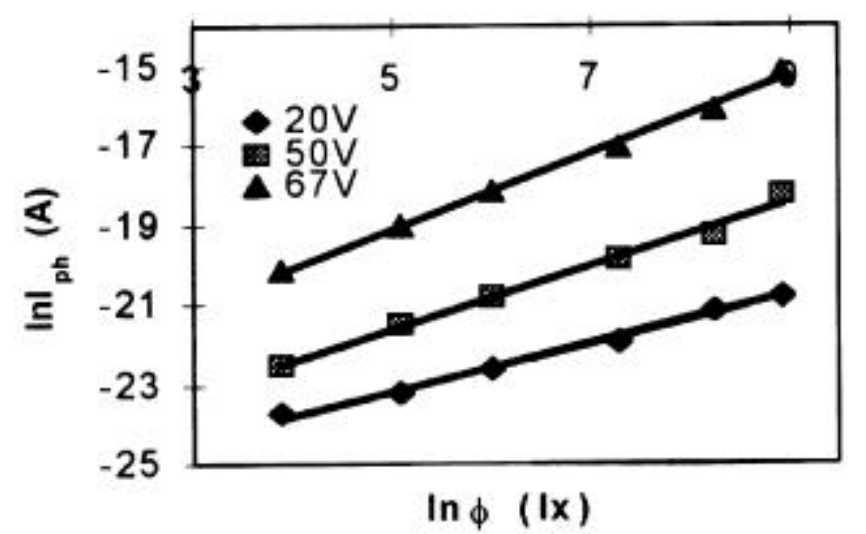

Figure 5. $\ln I_{\mathrm{ph}}-\ln \phi$ characteristics for CdSe film (C). Photocurrent $I_{\mathrm{ph}}=\left(I_{l}-I_{d}\right)$, here $I_{l}$ total current under illumination and $I_{d}$, dark current. 
Table 1. The calculated values of $l, v_{\mathrm{t}}, n_{0}, N_{\mathrm{t}}$ and $\theta$ for CdSe films.

\begin{tabular}{|c|c|c|c|c|c|c|c|}
\hline$t(\AA)$ & $T_{\mathrm{s}}(\mathrm{K})$ & Measured at & $V_{\mathrm{t}}(V)$ & $l$ & $n_{0}\left(\mathrm{~m}^{-3}\right)$ & $N_{\mathrm{t}}\left(\mathrm{m}^{-3}\right)$ & $\theta$ \\
\hline 850 & 303 & RT (303 K) & 20 & $1 \cdot 5$ & $5.47 \times 10^{21}$ & $2.85 \times 10^{27}$ & $7.72 \times 10^{6}$ \\
\hline 1400 & 473 & RT (303 K) & 30 & 1.08 & $1.90 \times 10^{22}$ & $5.44 \times 10^{27}$ & $1.78 \times 10^{7}$ \\
\hline \multirow[t]{4}{*}{2000} & 573 & RT (dark) & 50 & $2 \cdot 8$ & $6.06 \times 10^{21}$ & $1.04 \times 10^{27}$ & $4.27 \times 10^{6}$ \\
\hline & & 160 lx (w.1.) & 50 & $3 \cdot 9$ & $1.73 \times 10^{22}$ & $4.68 \times 10^{26}$ & $1.21 \times 10^{7}$ \\
\hline & & $15001 \mathrm{~lx}$ (w.1.) & 50 & $4 \cdot 2$ & $2.66 \times 10^{22}$ & $3.92 \times 10^{26}$ & $1.87 \times 10^{7}$ \\
\hline & & $76001 x$ (w.1.) & 50 & $7 \cdot 8$ & $2 \cdot 12 \times 10^{23}$ & $1.98 \times 10^{26}$ & $1.50 \times 10^{8}$ \\
\hline \multirow[t]{6}{*}{3000} & 423 & RT $(303 \mathrm{~K})$ & 97 & $2 \cdot 0$ & $3.73 \times 10^{16}$ & $2.17 \times 10^{30}$ & $10 \cdot 55$ \\
\hline & & $313 \mathrm{~K}$ & 97 & $2 \cdot 0$ & $6.67 \times 10^{16}$ & $1.68 \times 10^{30}$ & $19 \cdot 10$ \\
\hline & & $333 \mathrm{~K}$ & 97 & 2.63 & $1.94 \times 10^{17}$ & $1.56 \times 10^{29}$ & $56 \cdot 45$ \\
\hline & & $353 \mathrm{~K}$ & 97 & $2 \cdot 75$ & $6 \cdot 14 \times 10^{17}$ & $8 \cdot 16 \times 10^{28}$ & $1.78 \times 10^{2}$ \\
\hline & & $373 \mathrm{~K}$ & 97 & $2 \cdot 7$ & $1.75 \times 10^{18}$ & $6.38 \times 10^{28}$ & $5.09 \times 10^{2}$ \\
\hline & & $393 \mathrm{~K}$ & 97 & $2 \cdot 7$ & $5.02 \times 10^{18}$ & $4.38 \times 10^{28}$ & $14.6 \times 10^{2}$ \\
\hline
\end{tabular}

RT, room temperature; w.l., white light.

dangling bonds that give rise to large number of localized states. These localized states act as carrier trapping centres and after trapping the injected charge from electrodes they become charged and thereby expected to build up a space charge. This build up of space charge by inherent native defects then play a key role in determination of SCLC process. At low field, the rate of carrier injection is small and therefore the current is entirely governed by the intrinsic free charge carrier density. When the bias is applied beyond $V_{\mathrm{t}}$, the rate of injected charge density increases and exceeds the thermally generated free charge carrier density. As the ambient temperature is increased from room temperature, this consequently leads to an increase of probability of thermal ionization of trapping centres. Therefore, the probability of trapping of injected charge with increasing temperature decreases. $N_{\mathrm{t}}$ then decreases and there is an increase in $\theta$ with temperature. It is observed that trap depth decreases on increasing $T_{\mathrm{s}}$ and $t$ as shown for film (C) (table 2). At high $T_{\mathrm{s}}$ and $t$ the film grain size becomes higher which effectively reduces the inter grain boundary defect states and thereby decreases trap depth. The calculated values of the parameters and their varying nature are well in agreement with the results reported by other workers (Chan and Hill 1976; Buragohain and Barua 1987; Sathyalatha et al 1989).

The SCLC conduction under external illumination may also be due to the injected charge density from electrodes as in dark. When the film is illuminated by light, additional photo-excited carriers are generated in the films. The current under illumination at any temperature increases over dark owing to additional photogenerated majority carrier electrons as well as the contribution from barrier modulation by minority carrier holes. It is seen that the sublinear behaviour of photocurrent on light intensity for applied voltage around $V_{\mathrm{t}}$ (figure 5) clearly reveals the cooperation of traps and recombination centres (Rose 1956). In the presence of light the quasi fermi levels pull towards the respective band edge. The trapping centres
Table 2. Estimated trap depths found from 4 CdSe films.

\begin{tabular}{lcccc}
\hline Film & Bias applied $(\mathrm{V})$ & $E_{\mathrm{t}}(\mathrm{eV})$ & Light $(\mathrm{lx})$ & $E_{\mathrm{t}}(\mathrm{eV})$ \\
\hline A & 30 & $0 \cdot 53$ & & \\
B & 45 & $0 \cdot 60$ & & \\
C & 50 & $0 \cdot 51$ & 160 & $0 \cdot 48$ \\
& 67 & $0 \cdot 49$ & 1500 & $0 \cdot 40$ \\
& 90 & $0 \cdot 42$ & 7600 & $0 \cdot 37$ \\
D & 123 & $0 \cdot 58$ & & \\
\hline
\end{tabular}

then effectively act as recombination centres and recombine with the photogenerated minority carrier holes. This in turn reduces the grain boundary potential barrier heights and contribute to the enhancement of film conductivity (Kalita et al 1999). The total trap density and corresponding trap depth under illumination thus decreases owing to recombination with minority carriers.

\section{Conclusions}

The $I-V$ characteristics of CdSe thin films have been examined in dark at different temperatures and under illuminations. The $I-V$ curves show nearly ohmic behaviour at low bias and SCLC at high bias range. The transition voltage from ohmic to SCLC is independent of ambient temperature and intensity of illumination. The defect states in the films form space charge on trapping the injected charge from electrodes and thereby determine the SCLC process. A slope $>2$ in the high bias region confirms the SCLC governed by the exponential trap distribution preferably with deep traps. Trap depths are calculated within $0 \cdot 60-0 \cdot 37 \mathrm{eV}$. The ratio of injected charge to free charge, $\theta$, the free carrier electron density $\left(n_{0}\right)$ increase with the increase of ambient temperature and intensity of illumination whereas the total trap density, $N_{\mathrm{t}}$, decreases. A decrease in trap depth with increasing $T_{\mathrm{s}}$ and $t$ is also due to the reduction of total grain boundary trap density in the films. The defect controlled SCLC is 
also supported by the sublinear behaviour of $\ln I_{\mathrm{ph}}-\ln \phi$ characteristics in the films.

\section{References}

Bak G W and Lipinski A 1994 Thin Solid Films 238290

Buragohain M and Barua K 1987 Indian J. Phys. A61 559

Chan D S H and Hill A E 1976 Thin Solid Films 38163

Devi S and Prakash S G 1992 Indian J. Pure \& Appl. Phys. 3018

Devi S and Prakash S G 1993 Indian J. Pure \& Appl. Phys. 31161

El-Kadry N, Ashour A and Mahmoud S A 1995 Thin Solid Films 269112

Gogoi S and Barua K 1982 Thin Solid Films 92227

Gould R D and Ismail B S 1992 J. Mater. Sci. Lett. 11313

Goswami A 1996 Thin film fundamentals (New Delhi: New Age Int. Pub.) p. 342

Kalita P K, Sarma B K and Das H L 1999 Indian J. Pure \& Appl. Phys. 37885
Khare P K, Pandey R K and Jain P L 2000 Bull. Mater. Sci. 23 325

Lhermitte C, Carles D and Vautier C 1975 Thin Solid Films 28 269

Nesheva D 1996 Thin Solid Films 28051

Oduor A O and Gould R D 1995 Thin Solid Films 270387

Padmanabhasarma H, Subramanian V, Rangarajan V and Murli K R 1995 Bull. Mater. Sci. 18875

Pawlikowski J M 1990 Thin Solid Films 19039

Raoult F, Fortin B and Colin Y 1989 Thin Solid Films 182114

Rose A 1956 in Photoconductivity conference (eds) R G Breckenridge et al (New York: John Wiley \& Sons) p. 21

Sathyalatha K C, Uthanna S and Jayaramareddy P 1989 Thin Solid Films 174233

Sharma K C, Sharma R and Garg J C 1992 Jpn J. Appl. Phys. 31742

Yodogawa Y, Shimizu K and Kanamori H 1973 Jpn J. Appl. Phys. 12711 